\title{
Effect of substituents on phenazine derivatives by spectral studies
}

\author{
G. Thirunarayanan ${ }^{1 *}$, I. Muthuvel ${ }^{1}$, V. Sathiyendiran ${ }^{2}$ \\ ${ }^{1}$ Department of Chemistry, Annamalai University, Annamalainagar-608 002, India \\ ${ }^{2}$ Department of Chemistry, Sourashtra College, Madurai-625 004, India \\ *E-mail address: thirunarayanan.g.10313@annamalaiuniversity.ac.in, \\ drgtnarayanan@gmail.com
}

\begin{abstract}
A series of eleven substituted dipyrido[3,2-a; $\left.2^{\prime}, 3^{\prime}-\mathrm{c}\right]$ phenazine derivatives have been synthesized and examined their purities by literature method. The infrared and ${ }^{13} \mathrm{C}$ NMR spectral data of prepared phenazines were correlated with Hammett substituent constants, $\mathrm{F}$ and $\mathrm{R}$ parameters using single and multi-regression analysis. From the results of statistical analysis, the effect of substituents on the infrared frequencies $\left(v, \mathrm{~cm}^{-1}\right)$ and ${ }^{13} \mathrm{C} \mathrm{nmr}$ chemical shifts $(\delta, \mathrm{ppm})$ has been studied.
\end{abstract}

Keywords: Phenazines; IR spectra; ${ }^{13} \mathrm{C}$ NMR spectra; Spectral correlation; Hammett substituent constants; Swain-Lupton's parameter

\section{INTRODUCTION}

Phenazine and it derivatives are an important ligand for metal complex formation in inorganic chemistry[1]. Inorganic chemists paid much more attention for studying the complex formation[1], physico-chemical properties[2] and biological activities[3,4] of phenazine-metal complexes. Phenazine molecules possess part of quinoxaline moiety. Spectral studies of phenazine and it derivatives were made by Lue et al.[5]. Phenazine ligands were employed for the study of synthesis, enantiomeric resolution and binding interactions with calf thymus DNA of Cr(III) complex by Vasudevan et al.[6]. Interaction of $\mathrm{Ru}(\mathrm{II})$-phenazine complex with CT-DNA study was made by Santos et al.[7]. The crystal structure, DNA cleavage activity of phenazine-Cu(II) complex was studied by Begum et. al.[8]. The structure activity relationship of phototoxic Fe(III)-phenazine complex was reported by Saha et al.[9] This phenazine ligand was applied for synthesizing Ru(II) Light Switch complexes[3], and its DNA-Binding[3], photo cleavage[4], spectral properties[1], DNA-Non covalent cross linking with nano particles[10], excited state behaviours in presence of nucleic acids[2], electronic properties[11], DNA mediated energy transfer[12], electrochemical characterization[13], metal based inhibitor of NEDD8-activating enzyme[14], tuned cell membrane affinity[15], synthesis of poly ketones[16], and photo oxidations[1]. Nair et al., have studied the optical properties of phenazine-Ru complexes [17]. Qsar and qspr study was established with various compounds such as chalcones [18], pyrazolines[19], imines[20], flavones[21], pyrimidines[22], carboxamides[23], oxazines[24], epoxides[25], sulfonamides[26], acyl bromides[27], Tröger's bases[28], di-imines[29]and thiadiazoles[30]. 
Thirunarayanan and Sekar was studied the spectral qsar correlations in the pyrazoline derivatives [31]. The correlation study of infrared and ${ }^{13} \mathrm{C}-\mathrm{NMR}$ chemical shifts of aryl hydrazides with Hammett substituent constants and $\mathrm{F}$ and $\mathrm{R}$ parameters were established by Thirunarayanan et al.,[32] Mayavel et al., have investigated the spectral correlationslinearity of infrared and NMR spectral frequencies of carbazole imines with Hammett substituent constants, F and R parameters[33]. Sathiyendiran et al.[34] have prepared some 2oxopropy diazenyl benzoic acids and studied the spectral correlation. Recently Thirunarayanan et al have studied the spectral qsar study of phenazine derivatives[35]. There was no spectral correlation study was reported with phenazine molecules in the past. Therefore the author have taken efforts to prepare some substituted phenazines, recorded their infrared and ${ }^{13} \mathrm{C}$ NMR spectra for the spectral correlations study.

\section{EXPERMMENTAL}

\subsection{General}

Merck and Sigma-Aldrich branded chemicals were used in this present study. The infrared spectra of all phenazines were recorded in SHIMADUZ Fourier Transform IR spectrophotometer using $\mathrm{KBr}$ discs. The ${ }^{13} \mathrm{C}$ NMR spectra of all compounds have been recorded in BRUKER AV 400 type spectrometer, using $\mathrm{CDCl}_{3}$ as a solvent, $100 \mathrm{MHz}$ for ${ }^{13} \mathrm{C}$ NMR spectra, taking TMS as standard.

\subsection{Synthesis of substituted phenazine derivatives}

The substituted phenazines were synthesized and the purities of the compounds were examined by literature method [1, 29-31]. The general structure of the substituted phenazine is shown in Fig.1.<smiles>[X]c1ccc2nc3c4cccnc4c4ncccc4c3nc2c1</smiles>

$\mathrm{X}=\mathrm{H}, \mathrm{NH}_{2}, \mathrm{PhCO}, \mathrm{COOH}, \mathrm{Cl}, \mathrm{F}, \mathrm{CH}_{3}, \mathrm{NO}_{2}, \mathrm{SO}_{3} \mathrm{H}$

Fig. 1. General structure of substituted phenazines. 


\section{RESULTS AND DISCUSSION}

In the present study, the authors have investigated the correlation of infrared and ${ }^{13} \mathrm{C}$ NMR spectral data of 10- and 11-substituted phenazines with Hammett substituent constants, $\mathrm{F}$ and $\mathrm{R}$ parameters using single and multi-linear regression analysis. Present investigation compound structure was shown is Fig. 1. This phenazines are symmetric molecule without substituents. When introducing the substituents at 10 and $11^{\text {th }}$ position, the symmetry will disturbed. With respect to $\mathrm{C}_{15}=\mathrm{N}_{14}$ and $\mathrm{C}_{6}=\mathrm{N}_{7}$, the substituents attached in $11^{\text {th }}$ position was considered as meta- and para- positions. Within the considerations, the authors have performed the assigned spectral frequencies were correlated separately with respect to $\mathrm{C}_{6}=\mathrm{N}_{7}$ and correlations performed with respect to $\mathrm{C}_{15}=\mathrm{N}_{14}$ systems in the phenazines.

\subsection{Infrared spectral correlation}

The assigned the $\mathrm{C}=\mathrm{N}$ stretches $\left(\mathrm{cm}^{-1}\right)$ of the present investigation substituted phenazines were tabulated in Table 1. These data were correlated with Hammett substituent constants, $\mathrm{F}$ and $\mathrm{R}$ parameters [18-35]. In this correlation, the Hammett equation was employed as,

Table 1. The infrared $\mathrm{C}=\mathrm{N}$ stretches $\left(\mathrm{cm}^{-1}\right)$ and ${ }^{13} \mathrm{C}$ chemical shifts $(\mathrm{ppm})$ of substituted phenazines.

\begin{tabular}{|c|c|c|c|c|c|}
\hline Sl. No. & $\mathrm{X}$ & $v \mathrm{C}=\mathrm{N}$ & $\delta \mathrm{CN}_{\text {Pyr }}$ & $\delta \mathrm{CN}_{\text {Phena }}$ & $\delta \mathrm{C}_{\text {ipso }}$ \\
\hline 1 & $\mathrm{H}$ & 1577 & 149.54 & 153.85 & 130.87 \\
\hline 2 & $\mathrm{NH}_{2}$ & 1574 & 149.38 & 153.74 & 146.33 \\
\hline 3 & $\mathrm{PhCO}$ & 1548 & 149.41 & 153.82 & 138.14 \\
\hline 4 & $\mathrm{COOH}$ & 1569 & 149.45 & 153.88 & 132.46 \\
\hline 5 & $\mathrm{Cl}$ & 1578 & 149.62 & 153.89 & 143.75 \\
\hline 6 & $\mathrm{~F}$ & 1574 & 149.61 & 153.81 & 145.66 \\
\hline 7 & $\mathrm{CH}_{3}$ & 1569 & 149.31 & 153.71 & 138.04 \\
\hline 8 & $\mathrm{NO}_{2}$ & 1579 & 150.19 & 153.98 & 149.56 \\
\hline 9 & $\mathrm{SO}_{3} \mathrm{H}$ & 1566 & 149.63 & 153.76 & 146.36 \\
\hline
\end{tabular}

$$
v=\rho \sigma+v_{0}
$$

where $v_{\mathrm{o}}$ is the frequency for the parent member of the series.

The results of statistical analysis are tabulated in Table 2. From Table 2, the single parameter correlation of $v \mathrm{C}=\mathrm{N} m$ - substituted pattern phenazines gave satisfactory correlation coefficients with $\sigma, \sigma^{+}, \sigma_{\mathrm{I}}$ constants and $\mathrm{F}$ parameters. Hammett substituent constants, $\mathrm{F}$ and $\mathrm{R}$ parameters except fluoro- substituent for $\sigma_{\mathrm{I}}$ and $\mathrm{R}$ parameter excluding $\mathrm{PhCO}$ substituent. The Hammett $\sigma_{\mathrm{R}}$ constants and $\mathrm{R}$ parameter were failing in correlations. Similarly the single parameter correlation of these stretches of $p$ - substituted pattern phenazines gave satisfactory correlation with Hammett $\sigma_{\mathrm{R}}$ constants and F parameters. The Hammett $\sigma, \sigma^{+}, \sigma_{\mathrm{I}}$ constants and R parameters were failing in correlations. All correlations gave positive $\rho$ values. This meant that the normal substituent effect operates in all systems. The failure in correlation was due to the inability of predicting the substituent effects on the frequencies along with the resonance conjugative structure as shown in Fig. 2. 
Table 2. Results of statistical analysis of IR and ${ }^{13} \mathrm{C}$ NMR spectral values of substituted phenazines with Hammett $\sigma, \sigma^{+}, \sigma_{\mathrm{I}}, \sigma_{\mathrm{R}}$ constants, $\mathrm{F}$ and $\mathrm{R}$ parameters.

\begin{tabular}{|c|c|c|c|c|c|c|c|}
\hline Frequency & Constant & $\mathrm{r}$ & $\mathrm{I}$ & $\rho$ & $\mathrm{S}$ & $\mathrm{n}$ & Correlated derivatives \\
\hline \multicolumn{8}{|c|}{ Correlations performed with $p$-substitution pattern } \\
\hline \multirow[t]{6}{*}{$v \mathrm{C}=\mathrm{N}\left(\mathrm{cm}^{-1}\right)$} & $\sigma$ & 0.818 & 1570.36 & 2.499 & 9.73 & \multirow[t]{6}{*}{9} & \multirow{6}{*}{$\begin{array}{l}\mathrm{H},-\mathrm{NH}_{2},-\mathrm{COPh},-\mathrm{COOH}, \\
-\mathrm{Cl},-\mathrm{F}, \mathrm{CH}_{3},-\mathrm{NO}_{2},-\mathrm{SO}_{3} \mathrm{H}\end{array}$} \\
\hline & $\sigma^{+}$ & 0.823 & 1570.36 & 3.624 & 9.67 & & \\
\hline & $\sigma_{\mathrm{I}}$ & 0.815 & 1568.13 & 5.603 & 9.64 & & \\
\hline & $\sigma_{\mathrm{R}}$ & 0.913 & 1569.43 & 12.336 & 9.01 & & \\
\hline & $\mathrm{F}$ & 0.923 & 1567.77 & 8.203 & 9.45 & & \\
\hline & $\mathrm{R}$ & 0.801 & 1569.71 & 0.415 & 9.76 & & \\
\hline \multirow[t]{6}{*}{$\delta \mathrm{CN}_{\text {pyre }}(\mathrm{ppm})$} & $\sigma$ & 0.971 & 149.42 & 0.623 & 0.19 & \multirow[t]{6}{*}{9} & \multirow{6}{*}{$\begin{array}{l}\mathrm{H},-\mathrm{NH}_{2},-\mathrm{COPh},-\mathrm{COOH}, \\
-\mathrm{Cl},-\mathrm{F}, \mathrm{CH}_{3},-\mathrm{NO}_{2},-\mathrm{SO}_{3} \mathrm{H}\end{array}$} \\
\hline & $\sigma^{+}$ & 0.960 & 149.49 & 0.467 & 0.21 & & \\
\hline & $\sigma_{\mathrm{I}}$ & 0.974 & 149.37 & 0.764 & 0.18 & & \\
\hline & $\sigma_{R}$ & 0.907 & 149.57 & 0.252 & 0.26 & & \\
\hline & $\mathrm{F}$ & 0.964 & 149.38 & 0.603 & 0.21 & & \\
\hline & $\mathrm{R}$ & 0.914 & 149.58 & 0.143 & 0.27 & & \\
\hline \multirow[t]{6}{*}{$\delta \mathrm{CN}_{\text {phena }}(\mathrm{ppm})$} & $\sigma$ & 0.969 & 153.78 & 0.198 & 0.06 & \multirow[t]{6}{*}{9} & \multirow{6}{*}{$\begin{array}{l}\mathrm{H},-\mathrm{NH}_{2},-\mathrm{COPh},-\mathrm{COOH}, \\
-\mathrm{Cl},-\mathrm{F}, \mathrm{CH}_{3},-\mathrm{NO}_{2},-\mathrm{SO}_{3} \mathrm{H}\end{array}$} \\
\hline & $\sigma^{+}$ & 0.973 & 153.80 & 0.183 & 0.06 & & \\
\hline & $\sigma_{\mathrm{I}}$ & 0.972 & 153.76 & 0.245 & 0.06 & & \\
\hline & $\sigma_{R}$ & 0.814 & 153.83 & 0.053 & 0.09 & & \\
\hline & $\mathrm{F}$ & 0.974 & 153.76 & 0.229 & 0.06 & & \\
\hline & $\mathrm{R}$ & 0.812 & 153.83 & 0.039 & 0.09 & & \\
\hline \multirow[t]{6}{*}{$\delta \mathrm{C}_{\mathrm{ipso}}(\mathrm{ppm})$} & $\sigma$ & 0.905 & 144.87 & 1.723 & 10.19 & \multirow[t]{6}{*}{9} & \multirow{6}{*}{$\begin{array}{l}\mathrm{H},-\mathrm{NH}_{2},-\mathrm{COPh},-\mathrm{COOH}, \\
-\mathrm{Cl},-\mathrm{F}, \mathrm{CH}_{3},-\mathrm{NO}_{2},-\mathrm{SO}_{3} \mathrm{H}\end{array}$} \\
\hline & $\sigma^{+}$ & 0.913 & 145.11 & 4.052 & 10.09 & & \\
\hline & $\sigma_{I}$ & 0.905 & 138.88 & 19.168 & 8.78 & & \\
\hline & $\sigma_{R}$ & 0.942 & 143.97 & 17.66 & 9.21 & & \\
\hline & $\mathrm{F}$ & 0.957 & 138.23 & 19.945 & 8.32 & & \\
\hline & $\mathrm{R}$ & 0.969 & 142.84 & 25.202 & 7.31 & & \\
\hline \multicolumn{8}{|c|}{ Correlations performed with $m$-substitution pattern } \\
\hline \multirow[t]{6}{*}{$v \mathrm{C}=\mathrm{N}\left(\mathrm{cm}^{-1}\right)$} & $\sigma$ & 0.901 & 1568.69 & 4.109 & 9.70 & \multirow[t]{6}{*}{9} & \multirow{6}{*}{$\begin{array}{l}\mathrm{H},-\mathrm{NH}_{2},-\mathrm{COPh},-\mathrm{COOH}, \\
-\mathrm{Cl},-\mathrm{F}, \mathrm{CH}_{3},-\mathrm{NO}_{2},-\mathrm{SO}_{3} \mathrm{H}\end{array}$} \\
\hline & $\sigma^{+}$ & 0.900 & 1569.77 & 0.132 & 9.76 & & \\
\hline & $\sigma_{\mathrm{I}}$ & 0.908 & 1567.88 & 6.992 & 9.58 & & \\
\hline & $\sigma_{\mathrm{R}}$ & 0.881 & 1568.95 & 14.939 & 9.21 & & \\
\hline & $\mathrm{F}$ & 0.928 & 1567.79 & 8.869 & 9.37 & & \\
\hline & $\mathrm{R}$ & 0.871 & 1519.75 & 0.415 & 9.72 & & \\
\hline \multirow[t]{6}{*}{$\delta \mathrm{CN}_{\text {pyre }}(\mathrm{ppm})$} & $\sigma$ & 0.992 & 149.35 & 0.833 & 0.16 & \multirow[t]{6}{*}{9} & \multirow{6}{*}{$\begin{array}{l}\mathrm{H},-\mathrm{NH}_{2},-\mathrm{COPh},-\mathrm{COOH}, \\
-\mathrm{Cl},-\mathrm{F}, \mathrm{CH}_{3},-\mathrm{NO}_{2},-\mathrm{SO}_{3} \mathrm{H}\end{array}$} \\
\hline & $\sigma^{+}$ & 0.961 & 149.49 & 0.389 & 0.21 & & \\
\hline & $\sigma_{\mathrm{I}}$ & 0.917 & 149.38 & 0.763 & 0.18 & & \\
\hline & $\sigma_{\mathrm{R}}$ & 0.827 & 149.58 & 0.293 & 0.27 & & \\
\hline & $\mathrm{F}$ & 0.905 & 149.25 & 0.501 & 0.22 & & \\
\hline & $\mathrm{R}$ & 0.804 & 149.58 & 0.143 & 0.27 & & \\
\hline$\delta \mathrm{CN}_{\text {phena }}(\mathrm{ppm})$ & $\sigma$ & 0.981 & 153.75 & 0.277 & 0.05 & 9 & $\mathrm{H},-\mathrm{NH}_{2},-\mathrm{COPh},-\mathrm{COOH}$ \\
\hline & $\sigma^{+}$ & 0.984 & 153.79 & 0.175 & 0.04 & & $-\mathrm{Cl},-\mathrm{F}, \mathrm{CH}_{3},-\mathrm{NO}_{2},-\mathrm{SO}_{3} \mathrm{H}$ \\
\hline & $\sigma_{\mathrm{I}}$ & 0.981 & 153.78 & 0.274 & 0.05 & & \\
\hline & $\sigma_{\mathrm{R}}$ & 0.805 & 153.82 & 0.027 & 0.09 & & \\
\hline & $\mathrm{F}$ & 0.907 & 153.76 & 0.228 & 0.05 & & \\
\hline & $\mathrm{R}$ & 0.813 & 153.83 & 0.039 & 0.09 & & \\
\hline$\delta \mathrm{C}_{\text {ipso }}(\mathrm{ppm})$ & $\sigma$ & 0.911 & 142.87 & 6.734 & 9.23 & 9 & $\mathrm{H},-\mathrm{NH}_{2},-\mathrm{COPh},-\mathrm{COOH}$ \\
\hline & $\sigma^{+}$ & 0.901 & 143.47 & 5.067 & 9.96 & & $-\mathrm{Cl},-\mathrm{F}, \mathrm{CH}_{3},-\mathrm{NO}_{2},-\mathrm{SO}_{3} \mathrm{H}$ \\
\hline & $\sigma_{\mathrm{I}}$ & 0.905 & 139.25 & 11.268 & 8.83 & & \\
\hline & $\sigma_{R}$ & 0.915 & 142.25 & 28.791 & 8.31 & & \\
\hline & $\mathrm{F}$ & 0.950 & 139.32 & 17.711 & 8.49 & & \\
\hline & $\mathrm{R}$ & 0.969 & 142.84 & 25.531 & 7.31 & & \\
\hline
\end{tabular}


$\mathrm{r}=$ correlation coefficient; $\mathrm{I}=$ intercept; $\rho=$ slope; $\mathrm{s}=$ standard deviation; $\mathrm{n}=$ number of correlated derivatives

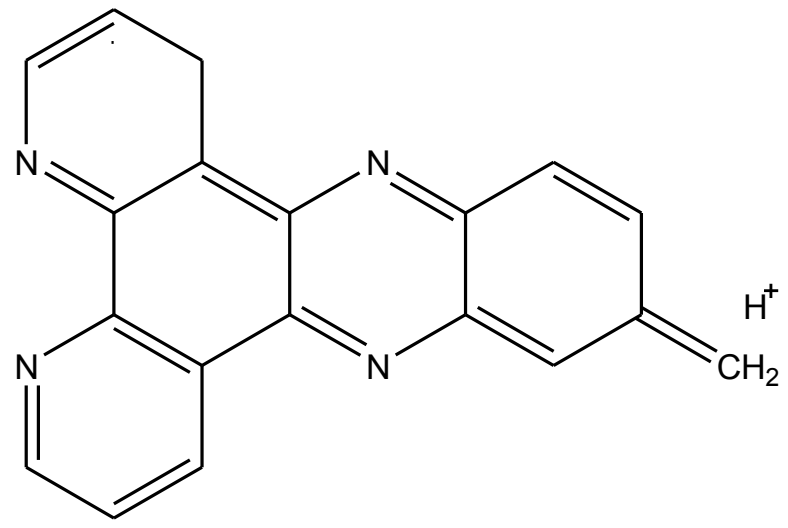

Fig. 2. The resonance-conjugative structure.

\section{2. ${ }^{13} \mathrm{C}$ NMR spectral correlation}

The ${ }^{13} \mathrm{C}$ NMR chemical shifts of $\mathrm{CN}$ pyridine and phenazines moieties were assigned and are presented in Table 1. These chemical shifts were correlated with Hammett substituent constants, $\mathrm{F}$ and $\mathrm{R}$ parameters using single and multi-regression analysis [18-35]. In these correlations, the Hammett equation was taken in the form as,

$$
\delta=\delta_{0}+\rho \sigma
$$

Where $\delta_{0}$ is the chemical shift of the corresponding parent compound.

The results of statistical analyses are shown in Table 2. The correlations performed with respect to $\mathrm{CN}$ ( $m$-substitution pattern), chemical shifts of $\delta \mathrm{C}=\mathrm{N}_{\text {pyri and phena }}(\mathrm{ppm})$ gave satisfactory correlation with $\sigma$ and $\sigma^{+}$and $\sigma_{\text {I }}$ constants $F$ parameters gave satisfactory correlations except PHCO substituent. The Ipso carbon chemical shifts gave satisfactory correlation with Hammett substituent constants, F and R parameters. All correlations gave positive $\rho$ values. These mean that the normal substituent effect operates in all systems. The reason for the failure in the correlation was already stated and along with the resonance conjugative structure as shown in Fig. 2.

The ipso carbon chemical shifts of the phenazines with Hammett $\sigma_{R}$ constant and $\mathrm{R}$ parameters gave satisfactory correlations. The Hammett $\sigma, \sigma^{+}, \sigma_{\mathrm{I}}$ constants and $\mathrm{F}$ parameter were fail in correlations. This is due to the inability of substituents along with conjugative structure as shown in Fig. 2.

The correlations performed with respect to $\mathrm{CN}$ ( $p$-substitution pattern), chemical shifts of $\delta \mathrm{C}=\mathrm{N}_{\text {pyri }}(\mathrm{ppm})$ gave satisfactory correlation Hammett substituent constants, $\mathrm{F}$ and $\mathrm{R}$ parameters excluding $\mathrm{PhCO}$ substituent. The correlation of $\delta \mathrm{C}=\mathrm{N}_{\text {phena }}(\mathrm{ppm})$ with Hammett $\sigma$ and $\sigma^{+}$and $\sigma_{I}$ constants $F$ parameters. The $\sigma_{R}$ and $R$ parameters gave poor correlations for this chemical shifts. All correlations gave positive $\rho$ values. These mean that the normal substituent effect operates in all systems. The reason for failure in correlation was already stated and it is associated with resonance conjugated structure as shown in Fig. 2. The Hammett gave satisfactory correlations except PHCO substituent. The Ipso carbon chemical shifts gave satisfactory correlation with Hammett substituent constants, F and R parameters. 
In single parameter correlations, some of the sigma constants gave poor correlations with were failed in correlations with Hammett substituent constants, $\mathrm{F}$ and $\mathrm{R}$ parameters. They are worthwhile when seeking multi-linear correlations with $\sigma_{I}$ and $\sigma_{R}$ constants or Swain Lupton's[36] $\mathrm{F}$ and $\mathrm{R}$ parameters gave satisfactory correlations for infrared and ${ }^{13} \mathrm{C}$ NMR spectral data of phenazines. The generated multi-regression analysis equations are shown in (3-26)

\section{Correlations performed with $\boldsymbol{m}$-substitution pattern}

$$
\begin{gathered}
v \mathrm{C}=\mathrm{N}\left(\mathrm{cm}^{-1}\right)=1567.71( \pm 5.071)+4.800( \pm 1.401) \sigma_{\mathrm{I}}+13.634( \pm 1.875) \sigma_{\mathrm{R}} \\
(R=0.933, \mathrm{n}=9, \mathrm{P}>90 \%) \\
v \mathrm{C}=\mathrm{N}\left(\mathrm{cm}^{-1}\right)=1567.76( \pm 4.832)+9240( \pm 1.248) \mathrm{F}+2.651( \pm 1.428) \mathrm{R} \\
(R=0.928, \mathrm{n}=9, \mathrm{P}>90 \%) \\
\delta \mathrm{CN}_{\text {Pyri }}(\mathrm{ppm})=149.36( \pm 0.085)+0.844( \pm 0.023) \sigma_{\mathrm{I}}+0.522( \pm 0.038) \sigma_{\mathrm{R}} \\
(R=0.982, \mathrm{n}=9, \mathrm{P}>95 \%)
\end{gathered}
$$

$$
\begin{gathered}
\delta \mathrm{C}_{\mathrm{ipso}}(\mathrm{ppm})=133.42( \pm 6.258)+15.023( \pm 1.472) \mathrm{F}-29.343( \pm 13.708) \mathrm{R} \\
(R=0.968, \mathrm{n}=10, \mathrm{P}>95 \%)
\end{gathered}
$$

\section{Correlations performed with $p$-substitution pattern}

$$
\begin{gathered}
v \mathrm{C}=\mathrm{N}\left(\mathrm{cm}^{-1}\right)=1567.90( \pm 5.234)+5.234( \pm 1.382) \sigma_{\mathrm{I}}+12.147( \pm 1.154) \sigma_{\mathrm{R}} \\
(R=0.934, \mathrm{n}=9, \mathrm{P}>90 \%) \\
v \mathrm{C}=\mathrm{N}\left(\mathrm{cm}^{-1}\right)=1567.71( \pm 5.299)+8.551( \pm 1.337) \mathrm{F}+1.621( \pm 0.143) \mathrm{R} \\
(R=0.968, \mathrm{n}=10, \mathrm{P}>95 \%) \\
\delta \mathrm{CN}_{\text {Pyra }}(\mathrm{ppm})=149.35( \pm 0.097)+0.772( \pm 0.253) \sigma_{\mathrm{I}}+0.279( \pm 0.028) \sigma_{\mathrm{R}} \\
(R=0.978, \mathrm{n}=9, \mathrm{P}>95 \%)
\end{gathered}
$$




$$
\begin{gathered}
\delta \mathrm{CN}_{\text {Pyra }}(\mathrm{ppm})=149.38( \pm 0.909)+0.667( \pm 0.271) \mathrm{F}+0.302( \pm 0.029) \mathrm{R} \\
(R=0.971, \mathrm{n}=9, \mathrm{P}>95 \%) \\
\delta \mathrm{CN}_{\text {Phena }}(\mathrm{ppm})=153.76( \pm 0.035)+0.243( \pm 0.092) \sigma_{\mathrm{I}}+0.044( \pm 0001) \sigma_{\mathrm{R}} \\
(R=0.973, \mathrm{n}=9, \mathrm{P}>95 \%) \\
\delta \mathrm{CN}_{\text {Phena }}(\mathrm{ppm})=153.76( \pm 0.054)+0.250( \pm 0.076) \mathrm{F}+0.099( \pm 0.008) \mathrm{R} \\
(R=0.980, \mathrm{n}=9, \mathrm{P}>95 \%) \\
\delta \mathrm{C}_{\text {ipso }}(\mathrm{ppm})=138.51( \pm 4.406)+18.662( \pm 1.637) \sigma_{\mathrm{I}}+16.998( \pm 1.272) \sigma_{\mathrm{R}} \\
(R=0.965, \mathrm{n}=9, \mathrm{P}>95 \%) \\
\delta \mathrm{C}_{\text {ipso }}(\mathrm{ppm})=138.29( \pm 3.267)+15.327( \pm 8.234) \mathrm{F}+21.850( \pm 8.741) \mathrm{R} \\
(R=0.982, \mathrm{n}=10, \mathrm{P}>92 \%)
\end{gathered}
$$

\section{CONCLUSIONS}

Totally nine substituted phenazines derivatives have been synthesized and their purities were examined by literature method. The infrared and ${ }^{13} \mathrm{C} N M R$ spectral frequencies of $\mathrm{C}=\mathrm{N}, \mathrm{C}-\mathrm{N}$ and ipso carbons of the phenazines were assigned and correlated based on $m$ - and $p$ - substituted system with Hammett substituent constants, $\mathrm{F}$ and $\mathrm{R}$ parameters using single and multi-regression analysis. Most of the correlations gave satisfactory correlation coefficients.

\section{References}

[1] B. Elias, C. Creely, G. W. Doorely, M. M. Feeney, C. Moucheron, A. K. DeMesmaeker, J. Dyer, D. C. Grills, M. W. George, P. Matousek, A. W. Parker, M, Towrie and J. M. Kelly, Chem. Eur. J., 14 (2008) 369-375

[2] W. Michal, A. J. Smith, S. Vasudevan, S. J. Quinn and J. M. Kelly, Phtochem. Photobiol Sci., 9 (2010)1196-1202

[3] S. Vidhisha, K. L. Reddy, Y. Praveen Kumar, M. Srijana and Satyanarayana, Int. J. Pharm. Sci. Rev. Res., 25 (2014) 197-205

[4] P. Naishadham and S. Satyanarayana, Int. J. Pharm. Sci. Rev., 4 (2013) 2265-2273

[5] J. G. Liu, B. H. Ye, H. Li, L. N. Ji, R. H. Li and J. Y. Zhou, J. Bioorg. Chem., 73(1999) 117-122

[6] S. Vasudevan, J. A. Smith, M. Wojdyla, R. McCabe, N. C. Fletcher, S. J. Quinn and J. M. Kelly, Dalton Trans, 39(2010) 3990-3998 
[7] T. M. Santos, J. Madureira, B. J. Goodfellow, M. G. B. Drew, J. P. Dejesus and V. Felix, Metal based Drugs., 8 (2001) 125-136.

[8] M.S. A. begum, S. Saha, A. Hussain and A. R. Chakravarthy, Indian J. Chem., 48A (2009) 9-14

[9] S. Saha, D. Mallick, R. Majumdar, M. Roy, R. R. Dighe, E. D. Jemmis and A. R. Chakravarthy, Inorg. Chem., 50 (2011) 2975-2987

[10] M. slim, N. Duristic, P.Grutter and H. F. Sleiman, ChemBioChem., 8(2007) 804-812.

[11] M. I. J. Palson, S. L. Howell, A. H. Flood, A. K. Burrell, A. G. Blackman and K. C. Gordon, Polyhedron., 23(2004)1427-1439

[12] M. Towric and A. W. Parker, S. P. Foxon and J. A. Thomas, Lasers for Science Facility Programme; Central Laser Facility Annual Report, (2005-2006)162-164.

[13] K. Maruyama, Y. Mishima, Y. Nakabayashi and J. Motonaka, Nucleic Acids Symposium Series, Oxford University Press, No. 44 (2000) 59-60

[14] H. J. Zhong, H. Yang, D. S. H. Chan, C. H. Leung, H. M. Wang, D. L. Ma, Plos One, 7(2012) e49574. doi:10.1371/journal.pone.0049574.

[15] F. R. Svensson, M. Matson, M. Li and P. Lincoln, Biophy. Chem., Accepted article, (2010); doi: 10.1016/j.bpc.2010.04.006

[16] D. Villagra, R. Lopez, S. A. Moya, C. Claver and A. Bastero, Organometallics., 27(2008) 1019-1021

[17] R. B. Nair, B. M. Kullum and C. J. Murphy, Inorg Chem., 36(1997) 962-965

[18] K. Sathiyamoorthy, V. Mala, S.P. Sakthinathan, D. Kamalakkannan, R. Suresh, G. Vanangamudi and G. Thirunarayanan, Spectrochim. Acta, 112A(2013) 245-256

[19] S. Sasikala, K. Thirumurthy, P. Mayavel and G. Thirunarayanan, Org. Med. Chem. Lett. (2012.) doi:10.1186/2191-2858-2-20.

[20] R. Suresh, D. Kamalakkannan, K. Ranganathan, R. Arulkumaran, R. Sundararajan, S.P. Sakthinathan, S. Vijayakumar, K. Sathiyamoorthy, V. Mala, G. Vanangamudi, K. Thirumurthy, P. Mayavel and G. Thirunarayanan, Spectrochim. Acta. 101A (2013) 239-248

[21] G. Thirunarayanan and K, G. Sekar, Int. Lett. Chem. Phys. Astro. 6 (2014) 39-47

[22] R. Arulkumaran, R. Sundararajan, V. Manikandan, V. Sathiyendiran, S. Pazhamalai, G. Thirunarayanan, Int. Lett. Chem. Phys. Astro, 19(1) (2014) 25-15

[23] K. G. Sekar and G. Thirunarayanan, Int. Lett. Chem. Phys. Astro. 8(2) (2013) 160-174

[24] G. Thirunarayanan, Int. J. Sci. Res. Know. 1(12) (2013) 480-596

[25] G. Thirunarayanan and G. Vanangamudi, Spectrochim Acta, 81A (2011) 390-396 
[26] K. G Sekar and G. Thirunarayanan, Int. Lett. Chem. Phys. Astro. 8(3) (2013) 249-258

[27] G. Thirunarayanan, Int. Lett. Chem. Phys. Astro. 9(2) (2013) 152-161

[28] G. Thirunarayanan and M. Suresh, Int. Lett. Chem. Phys. Astro. 4 (1) (2014) 1-11

[29] G. Thirunarayanan, Bull. Chem. Soc. Ethiop. 28(1) (2014)73-79

[30] G. Thirunarayanan, Int. Lett. Chem. Phys. Astro. 5 (2014) 89-98

[31] G. Thirunarayanan and K. G. Sekar, J. Taibah Univ. Sci. 8 (2014)124-136

[32] G. Thirunarayanan, K.G. Sekar and R. Lakshmi Narayanan, Int. Lett. Chem. Phys. Astro. 13(1) (2014) 88-94

[33] P. Mayavel, K. Thirumurthy, S. Dineshkumar, G. Thirunarayanan, Q-Science Connect. (2014) DOI: http://dx.doi.org/10.5339/connect.2014.10

[34] V. Sathiyendiran, K. G. Sekar, G. Thirunarayanan, R. Arulkumaran, R. Sundararajan, D. Kamalakkannan, R. Suresh, V. Manikanadan, R. Vijayakumar, G. Vanangamudi, Int. Lett. Chem. Phys. Astro. 11(1) (2014) 33-43

[35] G. Thirunarayanan, I. Muthuvel and V. Sathiyendiran, Int. Lett. Chem. Phys. Astro. 19(2) (2014) 198-207

[36] C. G. Swain and E. C. Lupton, Jr, J. Am. Chem. Sco. 90 (1968) 4328-4337 